with splenomegaly but, unexpectedly, bone marrow fibrosis and hypocholesterolaemia (total cholesterol concentration $2.4 \mathrm{mmol} /$, low density lipoprotein cholesterol concentration $1.5 \mathrm{mmol} / \mathrm{l}$ ). It seems to us that the common denominator in low proliferating disorders with hypocholesterolaemia -that is, hairy cell leukaemia, myelofibrosis, and Gaucher's disease-is splenomegaly with an expanded reticuloendothelial system. Acute myeloid leukaemia, in contrast, is a disease of rapidly proliferating tumour cells, in which hypocholesterolaemia is associated with tumour cell burden and increased degradation by tumour cells mediated by low density lipoprotein receptors.

GUNNAR JULIUSSON Consultant in haematology and internal medicin

Department of Haematology,

University Hospital,

S-581 85 Linköping,

Sweden

SIGURD VITOLS Consultant in clinical pharmacology

Department of Clinical Pharmacology,

Karolinska Hospital,

Karolinska Hospital,

Sweden

\section{Primary care in accident and emergency departments}

Outcomes of study cast doubt on sensitivity of triage decisions and triage criteria

EDrToR,-Jeremy Dale and colleagues highlight the difficult and unresolved problem of appropriate triage for patients attending accident and emergency departments with "primary care" type problems. ${ }^{1}$ We have had a primary care service in our accident and emergency department since February 1993, which we recently analysed. ${ }^{2}$

At St Mary's Hospital we developed a triage decision tree to help nurses differentiate between accident and emergncy and general practice patients. Those presenting with minor injuries considered to be unlikely to require radiography were channelled to see a general practitioner, while those likely to need a radiographic investigation were directed to an accident and emergency doctor. We think that it is appropriate to include this differentiation in the triage scheme because general practitioners are not specifically trained in interpreting $x$ ray films. In the community genera practitioners receive a radiologist's report of the investigation they have requested rather than the film itself. In contrast, doctors working in an accident and emergency department gain experience and specific training and feedback on interpreting $x$ ray films.

When the triage decision tree was used 9960 $(16.6 \%)$ of the 60000 patients presenting to the accident and emergency department of St Mary's Hospital were deemed appropriate for primary care, compared with the $40.9 \%$ in Dale and colleagues' study at King's College Hospital. In contrast to the study at King's, where $25 \cdot 5 \%$ of primary care patients and $46.7 \%$ of accident and emergency patients underwent radiography, only $152(15.8 \%)$ of 960 primary care patients in our study did.

We agree with the authors that some of the outcomes of this study cast doubt on the sensitivity of the triage decisions or perhaps the triage criteria themselves. We believe that patients with recent injury who are likely to require radiography are best treated by doctors trained in accident and emergency medicine.

JANE FOTHERGILI Consultan

R TOUQUET Consultan

P WARD

Department of Accident and Emergency Medicine,

Registrar

London W2 1NY
1 Dale J, Green J, Reid F, Glucksman E, Higgs R. Primary care in the accident and emergency department. 1 . Prospective identification of patients. BMF 1995;311:423-6. (12 August.)

2 Ward P, Huddy J, Hargreaves S, Touquet $R$, Hurley $J$ Fothergill J. Primary care in London: an evaluation of general practioners working in an inner city accident and of general department. $¥$ Accid Emerg Med (in press).

\section{Cost of employing general practitioners in department may outweigh savings}

EDrToR,-Jeremy Dale and colleagues state that important benefits in terms of resource allocation might result if general practitioners were employed in accident and emergency departments to manage patients with "primary care" type problems..' Although this may be the case at King's College Hospital, where the rate of patients attending with such problems is $41 \%$, in areas of Britain with lower primary care attendance rates the cost of employing general practitioners might outweigh any savings from reductions in investigations.

The accident and emergency department at the Royal Hospital, Wolverhampton, is in an inner city area and sees roughly 76000 new patients a year. A prospective study was undertaken between 12 July 1993 and 19 September 1993 to determine the size and epidemiology of the primary care problem. Primary care patients were defined as those whose condition could best be managed by a general practitioner (for example, those with ear or throat infections, urinary tract infections, or chronic back pain). Staff were trained so that consistency of categorisation was assured. A total of 13453 new patients were seen, $13184(98 \%)$ of whom had a general practitioner (compared with 91\% at King's College Hospital). Altogether 461 $(3.4 \%)$ patients were judged to have problems that would have been more appropriately managed by their general practitioner. Three hundred and twenty seven ( $71 \%$ ) primary care attenders presented on Monday to Friday, two thirds of them presenting during surgery hours. On average, therefore, the Royal Hospital saw seven primary care attenders a day, most of whom attended when access to their general practitioner was easiest. The cost of employing general practitioners to deal with such a small number of patients is unlikely to outweigh the cost of excess investigations.

Why two similar departments should have such a wide variation in the primary care attendance rate is unclear, but this comparison highlights the need to exercise caution when extrapolating from the experience of one department to that of another. We believe that the individual accident and emergency departments should analyse the size and epidemiology of the primary care "problem" locally before adopting expensive solutions.

DEREK P BURKE

Senior registrar, accident and emergency medicine Walsall Manor Hospital

Walsall

NAJAM RASHID

Senior registrar, accident and emergency medicine

Heartlands Hospital

Birmingham

1 Dale J, Green J, Reid F, Glucksman E, Higgs R. Primary care in the accident and emergency department. II. Comparison of 427-30. (12 August.)

\section{Parents practise triage for paediatric attendances}

EDTTOR,-We agree with Jeremy Dale and colleagues that there is considerable overlap between the conditions presenting to primary care and those presenting to accident and emergency departments. ${ }^{1}$ We would point out, however, that, like many other authors, Dale and colleagues have drawn conclusions using information collected from only one side of the interface between primary and secondary care.
Our own study of paediatric attendances in accident and emergency was also a prospective study but examined attendance and process measures in primary care simultaneously to provide a more complete picture of the use of health care services by this group of patients. ${ }^{2}$ Dale and colleagues point out that triage can be used to differentiate patients into primary care and accident and emergency groups. The results of our study suggest that parents already practise a form of triage before seeking medical help. For example, over six weeks 730 children from the study practice sought medical advice; of these, 629 presented initially to the general practitioner, who dealt with all but 25 without onward referral to the accident and emergency department (two of the 25 did not attend the department). One hundred and twenty seven practice patients were seen at the accident and emergency department, of whom 104 were referred by their parents. The patients taken direct to the accident and emergency department differed in several important respects from those who attended their general practitioner initially Of the 96 patients who consulted after injury, $74(77 \%)$ presented direct to the accident and emergency department, compared with 27 (4\%) of the 634 children who had not been injured $(P<0.0001)$. This finding is supported by another study of childhood injuries, which found that 102 $(85 \%)$ of 120 injured children were taken direct to an accident and emergency department by their parents. In addition, seven of eight children with fractures in our study were taken direct to the accident and emergency department by their parents. The median age of children who consulted their general practitioner first was 3 years, compared with a median of 6 years for those who presented to the accident and emergency department first.

We broadly agree with Dale and colleagues' conclusions, but we would emphasise the need for caution in interpreting studies that examine only one side of the interface since a distorted perspective may be obtained.

TERRY BRADLEY Principal in general practice BRIGID MCCANN
Trainee general practitioner

Health Centre

Northern Ireland

JOHN GLASGOW

Accident and Emergency Department,

Accident and Emergency Department,

Reyal Belfast Hospita

Consulta

Department of Epidemiology and Public Health

Queen's University,

Belfast BT7 INN

1 Dale J, Green J, Reid F, Glucksman E, Higgs R. Primary care in the accident and emergency department. I. Prospective identification of patients. BMF 1995;311:423-6. (12 August.)

2 Bradley T, McCann B, Glasgow J, Patterson C. A comparison of paediatric consultation patterns in general practice and the accident and emergency department. Ulster Med $\mathcal{1 9 9 5 ; 6 4 :}$ 51-7.

3 Carter YH, Jones PW. Accidents among children under five years old: a general practice based study in north Staffordshire. $\mathrm{Br}$ Gen Pract 1993;43:159-63.

\section{Causal link between low cholesterol and cancer is unlikely}

EDITOR,-Goya Wannamethee and colleagues report that very low total cholesterol concentrations $(<4.8 \mathrm{mmol} / \mathrm{l})$ are associated with an excess risk of cancer and other non-cardiovascular diseases, which seems to be due to preclinical cancer, chronic ill health, smoking, and heavy drinking. Yet even patients with clinically overt cancer rarely have such low cholesterol concentrations. We studied the cholesterol concentrations in 